\title{
The miRNA bantam regulates growth and tumorigenesis by repressing the cell cycle regulator tribbles
}

\author{
Stephan U Gerlach, Moritz Sander, Shilin Song, Héctor Herranz (1)
}

One of the fundamental issues in biology is understanding how organ size is controlled. Tissue growth has to be carefully regulated to generate well-functioning organs, and defects in growth control can result in tumor formation. The Hippo signaling pathway is a universal growth regulator and has been implicated in cancer. In Drosophila, the Hippo pathway acts through the miRNA bantam to regulate cell proliferation and apoptosis. Even though the bantam targets regulating apoptosis have been determined, the target genes controlling proliferation have not been identified thus far. In this study, we identify the gene tribbles as a direct bantam target gene. Tribbles limits cell proliferation by suppressing G2/M transition. We show that tribbles regulation by bantam is central in controlling tissue growth and tumorigenesis. We expand our study to other cell cycle regulators and show that deregulated G2/M transition can collaborate with oncogene activation driving tumor formation.

DOI 10.26508/Isa.201900381 | Received 13 March 2019 | Revised 15 July 2019 | Accepted 15 July 2019 | Published online 22 July 2019

\section{Introduction}

Tissue growth has to be precisely regulated to generate organs with correct size, shape, and function. However, how growth is controlled remains a fundamental question in biology. In most animals, growth is the result of the increase in the number of cells, which is determined by the rate of cell division and cell death (Conlon \& Raff, 1999). Deregulation in those processes can result in the formation of tumors (Hanahan \& Weinberg, 2011). The wing imaginal disc of Drosophila has proven to be a useful system to study tissue growth control and to model different aspects related to tumor formation and metastasis (Gonzalez, 2013; Hariharan, 2015; Herranz et al, 2016).

miRNAs are small noncoding RNAs that have emerged as central regulators in the expression and function of animal genomes. miRNAs regulate multiple processes in development, including tissue growth, stem cell development, hormone action, and the organization and function of the central nervous system (Carthew et al, 2017). In addition, miRNAs control the activity of oncogenes and tumor suppressors and, thus, play crucial roles in cancer initiation and disease progression (Esquela-Kerscher \& Slack, 2006). Understanding the roles of miRNAs in development and cancer requires the identification of the target genes responsible for their functions.

The Hippo tumor suppressor pathway is a universal growth regulator and has been implicated in cancer (Pan, 2010). In Drosophila, the Hippo pathway consists of a cascade of kinases, which function sequentially to restrict the nuclear localization of the growth-promoting transcriptional coactivator, Yorkie (Yki) (Huang et al, 2005). Yki regulates the expression of the miRNA bantam and bantam mediates its growthpromoting role (Nolo et al, 2006; Thompson \& Cohen, 2006). bantam was the first miRNA discovered in Drosophila. It was identified as an element that, when overexpressed in the wing, promotes tissue growth (Hipfner et al, 2002; Brennecke et al, 2003). bantam stimulates growth by inducing proliferation and inhibiting apoptosis (Brennecke et al, 2003). bantam can also cooperate with the oncogene epithelial growth factor receptor (EGFR) in the formation of neoplastic tumors (Herranz et al, 2012b). Years of extensive research have led to the identification of multiple bantam targets, including suppressor of cytokine signaling at 36E (Socs36E), head involution defective (hid), capicua (cic), meiotic P26 (mei-P26), and enabled (ena) (Brennecke et al, 2003; Herranz et al, 2010, 2012a, 2012b; Becam et al, 2011). However, the current knowledge of the bantam target genes remains incomplete because individual depletion of any of them fails to mimic the effect of bantam driving tissue growth.

Here, we identify the gene tribbles (trbl) as a direct bantam target gene that mediates its growth regulatory role. We find that trbl downregulation in the wing disc accelerates $\mathrm{G} 2 / \mathrm{M}$ progression in the cell cycle. We show that simultaneous depletion of the bantam targets trbl and the proapoptotic gene hid reproduces the effect of bantam, promoting growth. The results presented here determine the minimal combination of bantam target genes sufficient to mimic the effect of bantam in tissue growth. We provide evidence that Trbl not only mediates the bantam growth-promoting role but also limits the magnitude of tissue overgrowth induced by the proto-oncogene Yki. Finally, we find that depletion of trbl can drive tumorigenesis in different contexts. Taken together, these results demonstrate that, in some instances, Trbl can limit tumorigenesis in the imaginal epithelium. In summary, our study demonstrates that trbl is a crucial target mediating the growth-promoting role and oncogenic function of bantam.

Department of Cellular and Molecular Medicine, University of Copenhagen, Copenhagen, Denmark 


\section{Results}

\section{bantam represses trbl}

miRNAs are negative regulators of gene activity. They bind to complementary sites on target mRNAs to induce mRNA cleavage or to repress mRNA translation. Understanding the oncogenic and growth regulatory roles of bantam requires the identification of its target genes. Bioinformatic analysis predicted two bantam-binding sites in the $3^{\prime}$ UTR of the gene trbl (Fig 1A). trbl encodes a kinase-like protein that controls cell proliferation by regulating the stability of the cell cycle regulator, cdc25-stg (Mata et al, 2000).
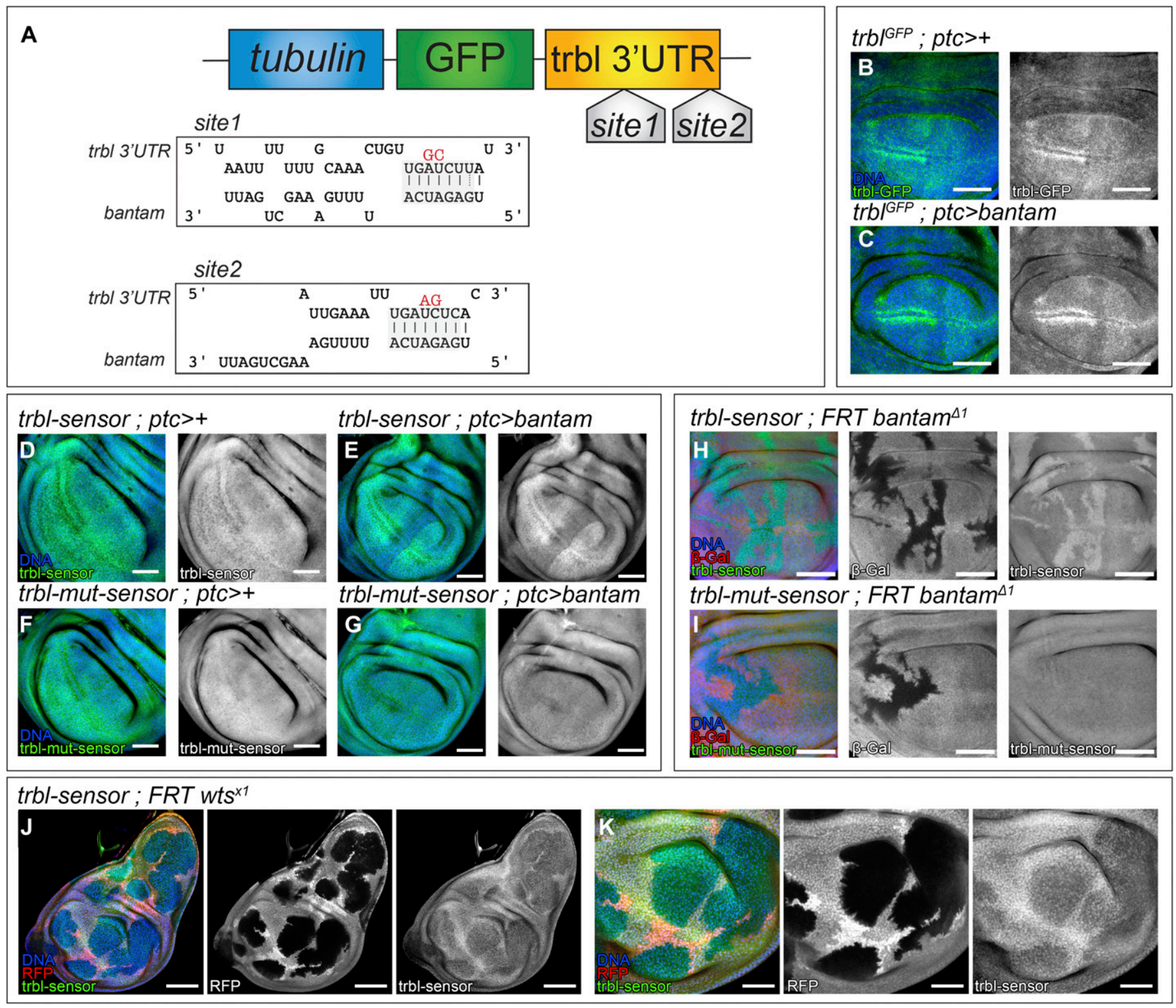

Figure 1. bantam represses trbl directly.

(A) Illustration of trbl-sensor showing predicted bantam-binding sites. The transgene consists of a tubulin promoter expressing the mRNA for GFP fused to the trbl $3^{\prime}$ UTR. The mRNA is sensitive to regulation through regulatory elements in the trbl $3^{\prime} U T R$. The trbl $3^{\prime}$ UTR includes two predicted binding sites for bantam (indicated as site 1 and 2). The predicted binding between the nucleotides of the trbl $3^{\prime} U T R$ and bantam is shown, and the predicted seed sequences are highlighted (connecting lines between the nucleotides and grey background). The trbl-mut-sensor has two nucleotides exchanged in each of the predicted seed sequences (highlighted in red). (B, C) Confocal micrographs of third-instar wing imaginal discs showing trbl-GFP expression (green and grey) in the genotypes indicated. DAPI was used to label the nuclei (blue). Larvae were kept constantly at $25^{\circ} \mathrm{C}$. Scale bar: $50 \mu \mathrm{m}$. (D-G) Confocal micrographs of third-instar wing imaginal discs showing the expression of trbl-sensor (D, E) and trbl-mut-sensor $(\mathrm{F}, \mathrm{G})$ in the genotypes indicated. The sensors are shown in green and grey. DAPI was used to label the nuclei (blue). Larvae were kept constantly at $25^{\circ} \mathrm{C}$. Scale bar: $50 \mu \mathrm{m}$. (H, I) bantam ${ }^{\Delta 1}$-mutant clones marked by the absence of $\beta$-Gal (red and grey), showing the expression of the trbl-sensor in (H) (green and grey), and showing the expression of the trbl-mut-sensor in (I) (green and grey). DAPI was used to label the nuclei (blue). To facilitate the analysis, the clones were induced in a Minute/+ background to allow the recovery of bigger clones. Scale bar: $50 \mu \mathrm{m}$. (J, K) wts ${ }^{x 1}$-mutant clones marked by the absence of RFP (red and grey), showing the expression of the trbl-sensor (green and grey). DAPI was used to label the nuclei (blue). (K) Shows a magnification of the wing disc shown in (J). Scale bar: $100 \mu \mathrm{m}$ in (J) and $50 \mu \mathrm{m}$ in (K). 
To validate a miRNA-target prediction, it is necessary to correlate the expression of miRNAs with those of their potential targets. miRNAs are posttranscriptional regulators and, to be functional, need to be co-expressed with their targets. To detect Trbl protein, we used a GFP-insertion in the trbl locus, resulting in a Trbl-GFP fusion protein (Mi(Mic)trbl-MI01025) (Venken et al, 2011). We monitored bantam expression by using a $P$ element insertion (bantam-lacZ) that reports expression from the bantam locus (Herranz et al, 2012a). Interestingly, Trbl and bantam were both observed in the wing disc, indicating that the bioinformatical prediction might reveal a functional interaction (Fig S1).

Next, we tested whether bantam up-regulation affected Trbl levels. Trbl showed an accumulation in two rows of cells abutting the dorsal-ventral boundary in the anterior compartment of the wing primordia (Fig 1B). Interestingly, Trbl limits G2/M progression (Mata et al, 2000) and the cells showing the highest Trbl levels correspond to the precursors of the sensory organs in the adult wing margin, which are arrested in G2 (Johnston \& Edgar, 1998). We made use of the binary Gal4:UAS system to manipulate bantam levels in the wing disc (Brand \& Perrimon, 1993). patched-Gal4 (ptcGal4) is expressed in a stripe of cells adjacent to the anteriorposterior and perpendicular to the dorsal-ventral border (Fig S1). Overexpression of UAS-bantam in the ptc domain reduced Trbl levels, as compared with the surrounding normal cells (Fig 1C). We obtained comparable results using the EP3622 element (Fig S1), an EP line inserted in the bantam locus that, when combined with a Gal4 driver, directs the expression of bantam (Hipfner et al, 2002). Consistent with those observations, Trbl-GFP levels were increased in bantam mutants (Fig S1). These results, coupled with the identification of bantam-binding sites in the trbl 3'UTR and the observations that trbl and bantam are co-expressed, suggest that trbl is a potential bantam target gene.

\section{trbl is a direct bantam target}

To assess whether bantam regulates trbl directly, we cloned the trbl 3'UTR downstream of a tubulin promoter-EGFP reporter plasmid (trbl-sensor, Fig 1A). The trbl-sensor was expressed ubiquitously in the wing imaginal disc (Fig 1D). Consistent with our previous observations, bantam overexpression in the ptc domain led to a robust reduction of the trbl-sensor (Fig 1E). To test whether trbl regulation by bantam occurred through the predicted binding sites, we generated a mutant version of the trbl-sensor (trbl-mut-sensor), carrying modifications in the both binding sites (illustrated in Fig 1A). Remarkably, the trbl-mut-sensor was not sensitive to bantam overexpression (Fig $1 \mathrm{~F}$ and $\mathrm{G}$ ). Overexpression of bantam using EPbantam (EP3622) yielded similar results, yet the regulation over the trbl-sensor was lower in magnitude than the one observed when expressing UAS-bantam (Fig S1).

Next, we studied whether the trbl 3'UTR was sensitive to endogenous bantam levels. To perform that analysis, we generated bantam null mutant clones (FRT bantam- $\Delta 1$ ) in the wing disc. bantam mutant clones grow poorly and are eliminated from the wing epithelium presumably by cell competition (Thompson \& Cohen, 2006). To bypass this issue, we provided the bantam mutant clones with a competitive advantage over neighboring cells by inducing the clones in a Minute/+ background (Morata \& Ripoll,
1975). The bantam mutant clones were marked by the absence of lacZ expression. bantam mutant cells up-regulated the trbl-sensor (Fig 1H). In contrast to that, the expression of the trbl-mut-sensor was not affected in bantam clones (Fig 11), providing additional evidence that trbl regulation by bantam is direct. The trbl-sensor was not affected in +/+ control clones induced in a Minute/+ background (Fig S2). In summary, these observations indicate that trbl is a direct bantam target gene and show that the trbl $3^{\prime}$ UTR is sensitive, not only to bantam overexpression but also to endogenous bantam.

The Hippo pathway regulates the expression of bantam, which is central in mediating the Yki growth regulatory role (Nolo et al, 2006; Thompson \& Cohen, 2006). Hippo activation results in Warts (Wts) phosphorylation, which acts with its cofactor Mats to phosphorylate and inactivate Yki. wts-mutant tissue up-regulates Yki and overgrows (reviewed in (Pan, 2010)). As observed in wing discs up-regulating bantam, wts mutant cells down-regulated the trbl-sensor (Fig 1) and K). The trbl 3'UTR is, thus, sensitive to Hippo pathway activity.

\section{trbl antagonizes G2/M transition in the wing disc}

The conserved gene trbl was first identified in Drosophila as a gene coordinating embryonic cell division and morphogenesis (Mata et al, 2000). In the wing disc, cells overexpressing trbl show longer G2/M phases and accumulate Cyclin E (Mata et al, 2000; Reis \& Edgar, 2004). However, the consequences of downregulating trbl in the wing disc and its effects in growth control and tumorigenesis are not yet determined.

The fluorescence ubiquitin cell cycle indicator method (FUCCI) is a two-color sensor that provides a readout of the cell cycle phase of each cell in a population (Sakaue-Sawano et al, 2008). Drosophila FUCCI (Fly-FUCCI) marks cells in the G1-phase in green, cells in the $\mathrm{S}$-phase (DNA synthesis) in red, and cells in the G2-phase in yellow (Fig 2A) (Zielke et al, 2014). We combined the use of Fly-FUCCI with flow cytometry-based cell cycle analysis to study the cell cycle dynamics in discs modulating trbl. Expression of UAS-Fly-FUCCI in normal discs showed cells labeled in red, green, and yellow, indicating the presence of proliferating cells (Fig 2B). trbl overexpression led to an increase in cells labeled in yellow, representing an accumulation of cells in G2 (Fig 2C). We used anti-phosphoHistone $\mathrm{H} 3(\mathrm{PH} 3)$ as a specific marker of cells in mitosis to study whether trbl overexpression affected the rate of cell proliferation of the wing disc. We detected a reduction in the number of $\mathrm{PH} 3$-positive cells in discs expressing UAS-trbl when compared with control discs (Fig 2E-G). These observations are consistent with previous reports (Mata et al, 2000; Reis \& Edgar, 2004) and show that trbl overexpression limits cell proliferation by dampening $\mathrm{G} 2 / \mathrm{M}$ progression.

Next, we evaluated the role of endogenous trbl in the wing disc. Depletion of trbl by expression of a UAS-trbl-RNAi transgene caused a reduction in the number of cells marked in yellow, which indicates that those cells show faster G2/M transition (Fig 2D). The efficiency of the UAS-trbl-RNAi transgene is shown in Fig S3. In sum, these results confirm previous observation and reveal that endogenous trbl limits G2/M progression in the wing disc.

We used the adult wing to analyze how trbl affected cell number and tissue size. In the adult wing, each hair-like structure corresponds 

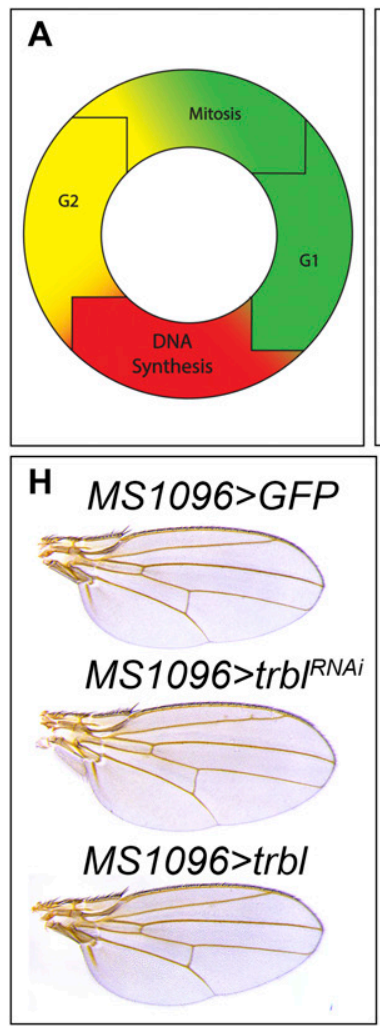
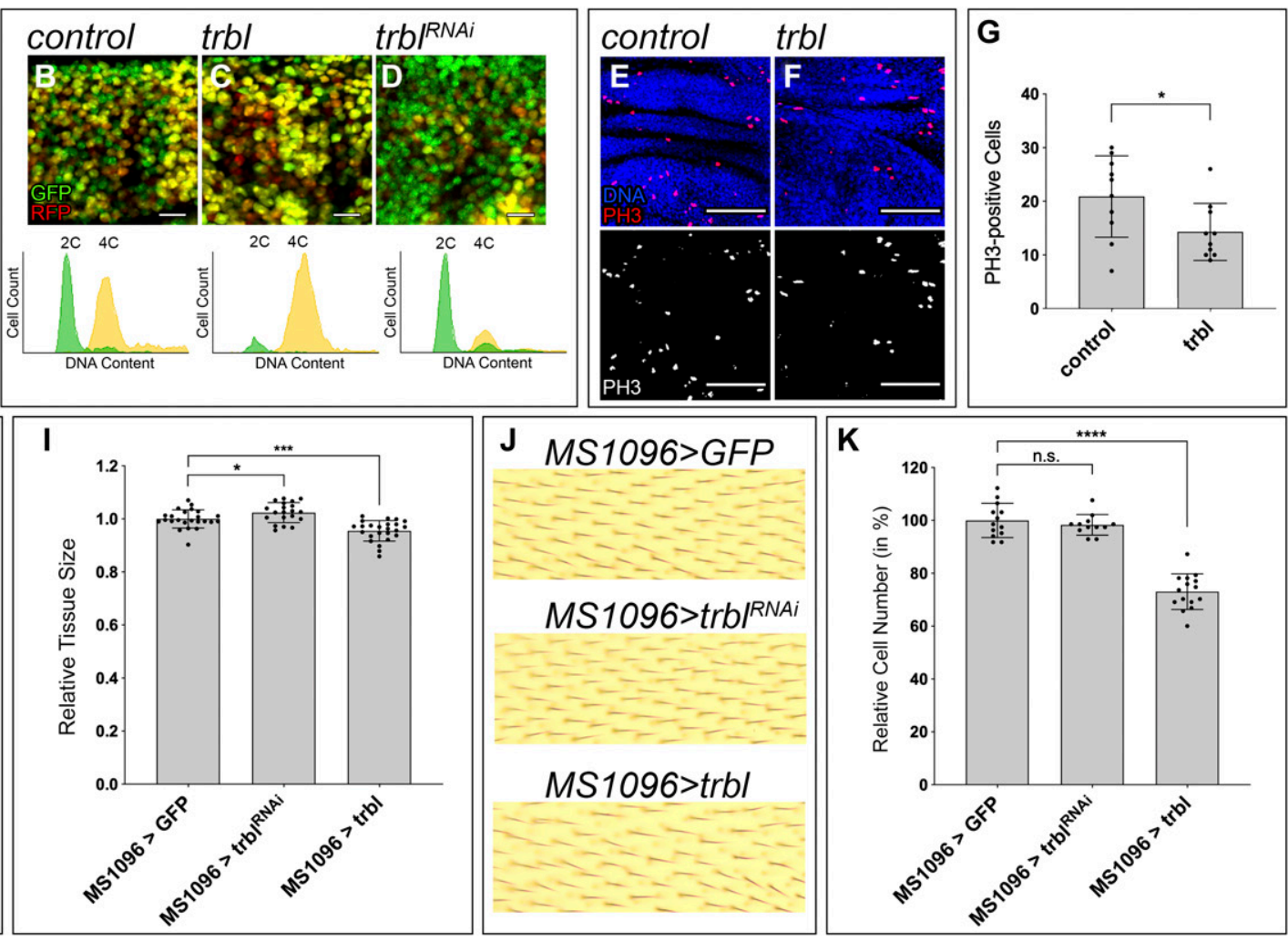

$\mathbf{K}$

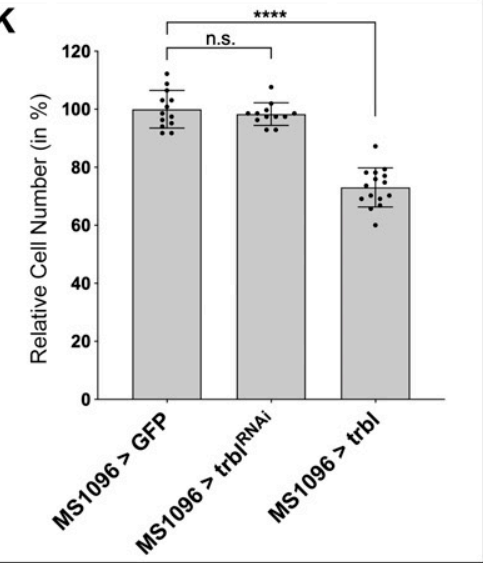

Figure 2. trbl phenotype in the wing disc.

(A) Illustration of fly-FUCCI system. Cells are labeled in green in late mitosis and the G1 phase, in red during the S-phase (DNA synthesis), and in yellow in the G2 phase and early mitosis. (B-D) Third-instar wing imaginal discs expressing the fly-FUCCI system together with UAS-trbl (C) and UAS-trbl-RNAi (D). The indicated genes were expressed under the control of the ap-Gal4 driver. ap-Gal4:UAS-FUCCI was used as control (B). Scale bar: $10 \mu \mathrm{m}$. Bottom panels show flow cytometry cell cycle analysis by quantification of DNA content in the genetic backgrounds shown in (B-D). Flow cytometry and fly-FUCCI were combined and the corresponding profiles are shown in the bottom of each panel. In these profiles, 2 C and 4C DNA contents are indicated, and the cells in G1 (green) and G2 (yellow) are highlighted. (E, F) Confocal micrographs of third-instar wing imaginal discs showing mitotic cells labeled by anti-PH3 (red and grey) in the following genotypes: ap-Gal4:UAS-GFP (E) and ap-Gal4:UAS-GFP; UAS-trbl (F). DAPI was used to label the nuclei (blue). Scale bars: $50 \mu \mathrm{m}$. (G) Quantification of PH3-positive cells in third-instar wing imaginal discs. Examples of quantified area in (E, F). Statistical significance was determined by unpaired $t$ test $\left(n=10\right.$; $\left.{ }^{*} P<0.05\right)$. (H) Cuticle preparations of adult wings of the genotypes indicated. (I) Quantification of adult wing size. Wing area is normalized to the mean of the control. Statistical significance was determined by unpaired $t$ test $(n=24$ [MS1096 $>$ GFP], $n=20$ [MS1096>trblRNAi], $n=24$ [MS1096>trbl]; $\left.{ }^{*} P<0.05 ;{ }^{* *} P<0.001\right)$. (J) High-magnification image showing cuticle preparations of adult wings of the genotypes indicated. Note the reduction in cell density reflecting the presence of bigger cells in the discs overexpressing trbl (MS1096>trbl). (K) Quantification of cell number in adult wings. Examples of quantified area in (J). Cell number is normalized to the mean of the control. Statistical significance was determined by unpaired $t$ test ( $\mathrm{n}=13$ [MS1096>GFP], $\mathrm{n}=12$ [MS1096>trbl-RNAi], $\mathrm{n}=15$ [MS1096>trbl]; ns, not significant; $\left.{ }^{* * * *} \mathrm{P}<0.0001\right)$.

to a trichome originating from a single epithelial cell. Hence, trichome density can be used as a proxy of cell size and number. trbl depletion did not affect cell or wing size. In contrast, trbl overexpression caused a mild reduction in wing size. These wings showed an increase in cell size revealed by reduced trichome density, indicating that trbl-overexpressing wings had fewer cells (Figs $2 \mathrm{H}-\mathrm{K}$ and $\mathrm{S4}$ ). The increased cell size observed upon trbl upregulation is characteristic of cells with delayed cell cycle progression (Neufeld et al, 1998) and is consistent with the $\mathrm{PH} 3$ staining and the FUCCI-FACS analysis.

\section{trbl is involved in the growth regulatory role of bantam}

The fact that Trbl regulates the cell cycle in the wing disc indicates that this gene is a promising candidate mediating the role of bantam, inducing cell proliferation. If trbl repression by bantam contributes to the growth-promoting role of this miRNA, we reasoned that restoring trbl expression in discs expressing bantam should reduce the magnitude of tissue overgrowth. Although trbl overexpression did not overtly affect the size of the wing disc, bantam-induced overgrowth was reduced by expression of UAStrbl in place of the UAS-lacZ control (Fig 3A-D and G). Taken together with the finding that bantam regulates trbl, these results suggest that bantam acts, at least partially, through trbl to stimulate tissue growth.

Given that bantam is an essential Yki target mediating its growth regulatory role (Nolo et al, 2006; Thompson \& Cohen, 2006), we reasoned that Trbl should also restrict the growth-promoting effect of Yki. Indeed, co-expression of trbl with yki was sufficient to limit the magnitude of tissue overgrowth induced by yki on its own (Fig $3 \mathrm{E}-\mathrm{G})$. It is worth noting that trbl expression in an otherwise normal background did not have a strong impact on the size of the wing 

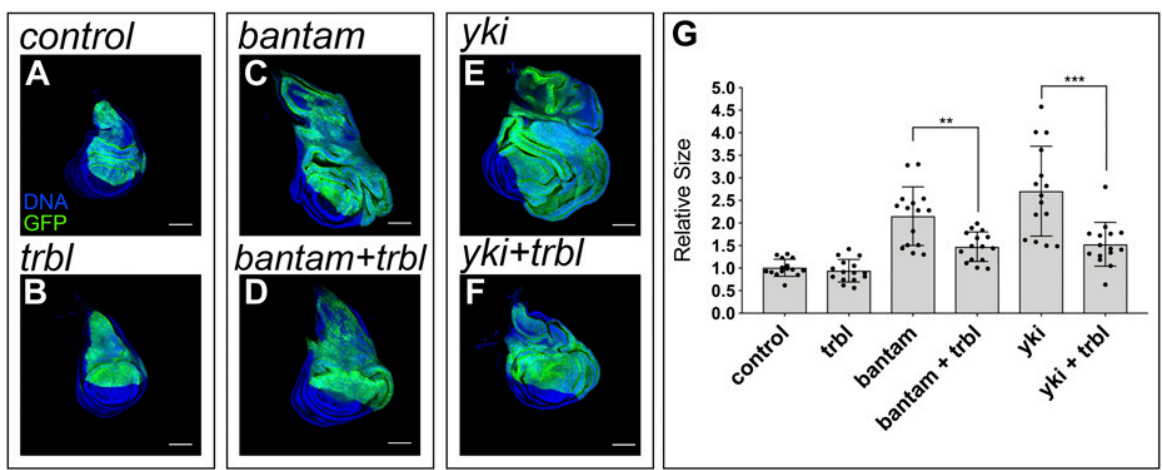

Figure 3. trbl dampens bantam- and Yki-induced tissue growth.

(A-F) Confocal micrographs of third-instar wing imaginal discs. The indicated genes were expressed under the control of the ap-Gal4 driver (dorsal compartment). The discs also expressed UAS-GFP to label the region where the corresponding transgenes were induced (green). DAPI was used to label the nuclei (blue). ap-Gal4:UAS-GFP was used as control (A). Scale bar: $100 \mu \mathrm{m}$. (G) Quantification of GFP-positive area in third-instar wing imaginal discs. GFP-positive area is normalized to the mean of the control.

Statistical significance was determined by unpaired $t$ test $\left(n=15 ;{ }^{* *} P<0.01 ;{ }^{* * *} P<0.001\right)$.

imaginal tissue (Fig 3B and G), indicating that the growth-repressing function of trbl is specific to the Yki and bantam axis, and not a general consequence of trbl overexpression on normal tissue growth.

\section{bantam promotes tissue growth through trbl and hid}

The results shown previously suggest that trbl is a central bantam target in growth control. Although trbl knock down in the wing disc accelerated G2/M progression, it did not result in obvious tissue overgrowth (Fig $4 \mathrm{~A}, \mathrm{~B}$, and $\mathrm{E}$ ). This suggests that trbl is not the only gene through which bantam induces tissue overgrowth. Changes in cell proliferation in the imaginal discs are typically associated with an increase in cell death (Karim \& Rubin, 1998; Neufeld et al, 1998). In good agreement, the use of an antibody recognizing the activated form of caspase 3 revealed the presence of apoptotic cells upon trbl depletion (Fig 4F-H). bantam inhibits cell death by repressing the proapoptotic gene hid (Brennecke et al, 2003). As in the case of trbl, hid down-regulation did not affect the size of the wing disc (Fig $4 \mathrm{C}$ and E).

We have shown that independent depletion of the bantam targets trbl or hid did not mimic the bantam gain of function phenotype. A cooperative interaction between both genes might mediate the growth-promoting role of bantam. We hypothesized that, to promote overall tissue growth, bantam could use a dual mechanism: on the one hand, it would accelerate G2/M transition by repressing trbl and, on the other hand, it would inhibit apoptosis by repressing the proapoptotic gene hid. If this is true, replacing bantam expression by co-depletion of both targets should lead to a similar outcome. To test this hypothesis, we expressed simultaneously UAS-RNAi transgenes directed against both bantam targets: UAS-trbl-RNAi and UAS-hid-RNAi. Remarkably, concurrent depletion of trbl and hid led to the formation of overgrown imaginal discs (Fig 4D and E), resembling bantam overexpression. hid depletion did not completely rescue the induction of apoptosis observed in wing discs expressing UAStrbl-RNAi (Fig S5), suggesting that other proapoptotic genes, in addition to hid, might contribute to the apoptotic response to trbl knock down.

Next, we studied the consequences of down-regulating trbl and hid in discs with reduced bantam. miRNA sponges controlled by UAS sequences allow spatially controlled down-regulation of
miRNAs under Gal4 control (Loya et al, 2009). The expression of a UAS-bantam-sponge transgene causes a reduction in tissue size (Herranz et al, 2012a). Notably, discs expressing simultaneously UAS-bantam-sponge, UAS-trbl-RNAi, and UAS-hid-RNAi were increased in size (Fig $4 \mid-K)$. This suggests that co-depletion of trbl and hid is sufficient to drive tissue overgrowth, even when bantam levels are reduced.

We have shown that trbl and hid are important targets contributing to the growth-promoting role of bantam. Tumor formation appears to require both an increase in cell division and a suppression of apoptosis (Green \& Evan, 2002). The apoptotic response observed in wing discs depleting trbl might serve as a homeostatic process to regulate organ size and prevent the formation of tissue hyperplasia. To assess that, we expressed the baculovirus protein p35 (Hay et al, 1994) or a miRNA that inhibits the proapoptotic genes reaper, hid, and grim ( $m i R-R H G$ ) (Siegrist et al, 2010), as alternative means to repress apoptosis. Remarkably, suppression of apoptosis in cells depleting trbl also led to tissue overgrowth (Fig S5). The use of an independent UAS-trbl-RNAi line produced comparable results (Fig S5). Similar results were obtained when we overexpressed cdc25-stg as an alternative method of forcing G2/M transition (Fig S6). In sum, these results suggest that apoptosis in response to trbl depletion can be used as a homeostatic mechanism to offset tissue overgrowth.

Next, we studied cell proliferation in discs co-expressing p35 and trbl-RNAi. We used proliferation markers such as 5-ethynyl20-deoxyuridine (EdU), which marks cells in the S-phase and $\mathrm{PH} 3$ to label cells in mitosis. This revealed an increase in the cell division rate in cells depleting trbl and expressing p35, as compared with the normal cells in the ventral compartment (GFPnegative) that served as internal control (Fig S5). In conclusion, these results reflect that simultaneous induction of $\mathrm{G} 2 / \mathrm{M}$ and suppression of apoptosis causes tissue hyperproliferation and hyperplasia.

\section{Trbl suppresses the oncogenic function of bantam}

EGFR is a well-recognized cancer driver. Overexpression of wildtype EGFR in Drosophila causes the activation of the MAPK pathway and tissue hyperplasia (Herranz et al, 2012a). Cooperating factors are required for the development of neoplastic tumors. bantam has been identified as one of such factors, whereby bantam inhibits the 

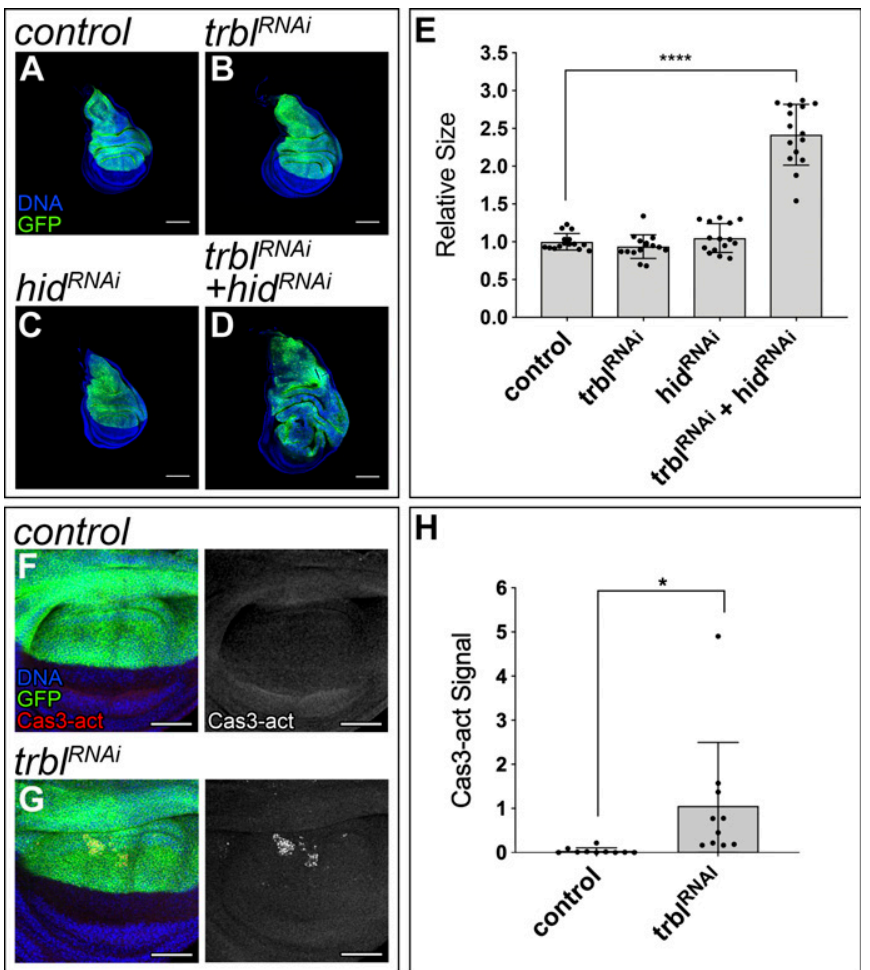

H
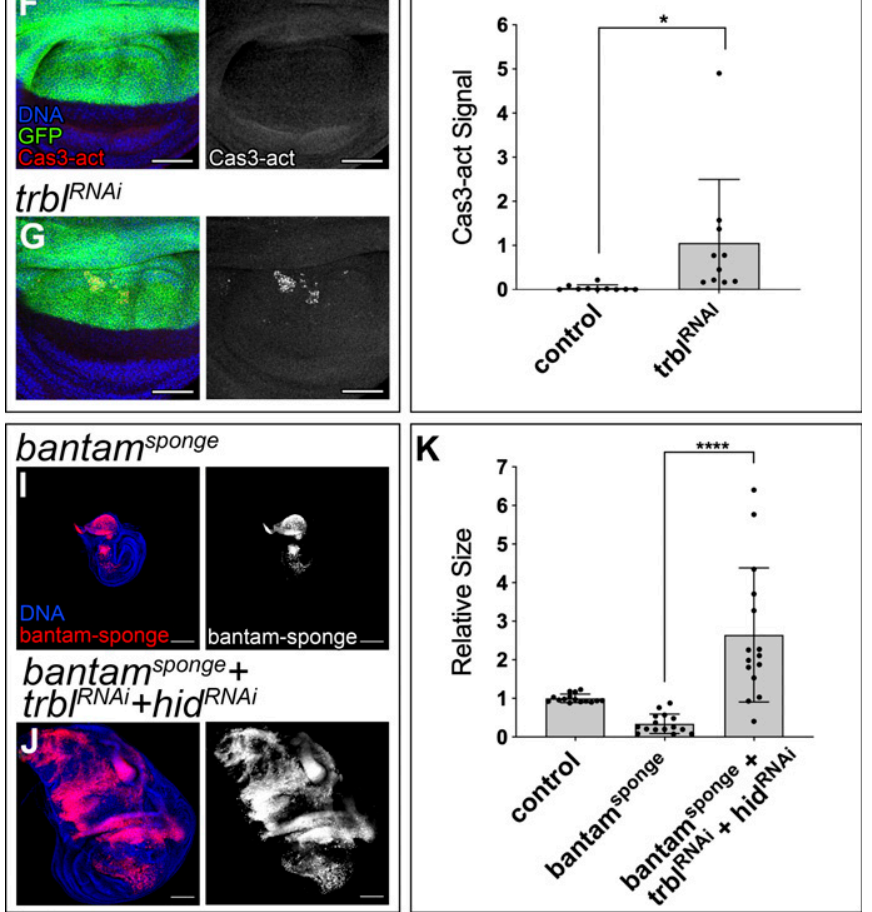

Figure 4. trbl and hid are key bantam targets in growth control.

(A-D) Confocal micrographs of third-instar wing imaginal discs. The indicated genes were expressed under the control of the ap-Gal4 driver (dorsal compartment). The discs also expressed UAS-GFP to label the region where the corresponding transgenes were induced (green). DAPI was used to label the nuclei (blue). ap-Gal4:UAS-GFP was used as control (A). Scale bar: $100 \mu \mathrm{m}$. (E) Quantification of GFP-positive area in third-instar wing imaginal discs. GFPpositive area is normalized to the mean of the control. Statistical significance was determined by unpaired $t$ test $\left(n=15 ;{ }^{* * * *} P<0.0001\right)$. $(\mathbf{F}, \mathbf{G})$ Third-instar wing imaginal discs showing staining against Cas3-activated signals (red and grey). DAPI was used to label the nuclei (blue). The indicated genes were expressed under the control of the ap-Gal4 driver (dorsal compartment). The discs also expressed UAS-GFP to label the region where the corresponding transgenes were induced (green). ap-Gal4:UAS-GFP was used as control (F). Larvae were kept constantly at $25^{\circ} \mathrm{C}$. Scale bar: $50 \mu \mathrm{m}$. (H) Quantification of Cas3-activated signal in the wing pouch of third-instar wing imaginal discs. The signal is represented by the mean grey value. Statistical significance was determined by unpaired $t$ test ( $n=10$; $\left.{ }^{*} P<0.05\right)$. Larvae were kept constantly at $25^{\circ} \mathrm{C}$. (I, J) Third-instar wing imaginal discs expressing UAS-bantam-sponge-RFP (I) and UAS-bantam-spongeRFP, UAS-trbl-RNAi, and UAS-hid-RNAi (J), under the control of the ap-Gal4 driver (dorsal compartment). Cells expressing bantam-sponge-RFP are labeled in red. DAPI was used to label the nuclei (blue). Larvae were kept constantly at $25^{\circ} \mathrm{C}$. Scale bar: $100 \mu \mathrm{m}$. (K) Quantification of the dorsal compartment size in thirdinstar wing imaginal discs. The control expressed GFP under the control of ap- suppressor of cytokine signaling Socs36E, which limits JAK/STAT signaling and tumor development (Herranz et al, 2012b).

We studied whether trbl also contributed to the bantam oncogenic function. Indeed, trbl expression in EGFR + bantam tumors impaired tumor growth leading to the formation of discs comparable in size with the one observed in discs expressing EGFR on its own (Fig $5 \mathrm{~A}-\mathrm{C}$ and $\mathrm{G}$ ). This suggests that trbl not only limits the bantam growth-promoting role, but also reduces its oncogenic effect. Next, we analyzed whether trbl down-regulation is sufficient to cooperate with the oncogene EGFR in tumorigenesis. Interestingly, although trbl depletion did not have a big impact in normal growth, trbl knock down in a context of EGFR overexpression fueled tumor growth (Fig 5D and G). These observations indicate that trbl behaves as a tumor suppressor in a context of EGFR expression.

Next, we studied the consequences of down-regulating other G2/ $M$ repressors in a context of EGFR activation. Activation of the Cdk1-Cyclin B complex induces cells to enter mitosis from G2. Myt1 is the main Cdk1 inhibitory kinase in the wing imaginal disc (Jin et al, 2008). Consistent with our observations in cells knocking down trbl, the expression of UAS-Myt1-RNAi accelerated G2/M transition and did not have an overt effect in disc size (Fig S7). Importantly, Myt1 depletion cooperated with EGFR in tumor formation (Fig 5E and G). Similar results were obtained when we drove G2/M transition by expressing cdc25-stg in discs up-regulating EGFR (Fig 5F and G). Together, this reveals that although deregulation in $\mathrm{G} 2 / \mathrm{M}$ transition does not have a major impact in normal tissue growth, it can be sufficient to trigger tumorigenesis in combination with the oncogene EGFR.

Trbl, in addition to regulate cell cycle progression, limits growth by antagonizing the insulin receptor/phosphatidylinositol 3-kinase (PI3K)/AKT protein kinase pathway (Das et al, 2014; Hong et al, 2016). This signaling cascade is hyperactivated in a wide range of human cancers (Vivanco \& Sawyers, 2002). Even though inducing G2/M appears to be sufficient to cooperate with EGFR in tumorigenesis (Fig 5D-G), we cannot rule out that PI3K/AKT regulation by trbl might also contribute to its tumor suppressor function.

\section{Trbl tumor suppressive role in other tumor contexts}

Cytokinesis failure leads to the formation of tetraploid cells. This generates an unstable situation that can lead to aneuploidy and tumor formation after cell division (Ganem et al, 2007). We have recently developed a genetic model in the wing imaginal disc of Drosophila to study the oncogenic potential of cytokinesis failure. The Drosophila gene peanut (pnut) belongs to the Septin family of proteins and is required for cytokinesis (Neufeld \& Rubin, 1994). RNAi-mediated depletion of pnut in the wing disc causes cytokinesis failure that leads to apoptosis and growth defects. We have reported that the oncogene yki is able to induce tumorigenesis in

Gal4 to mark the dorsal compartment. The RFP area was used to determine the size of the dorsal compartment for "bantam-sponge" and "bantam-sponge+trblRNAi+hid-RNAi." Sizes of the dorsal compartment are normalized to the mean of the control. Statistical significance was determined by unpaired $t$ test ( $n=15$; $\left.{ }^{* * * *} P<0.0001\right)$. Larvae were kept constantly at $25^{\circ} \mathrm{C}$. 


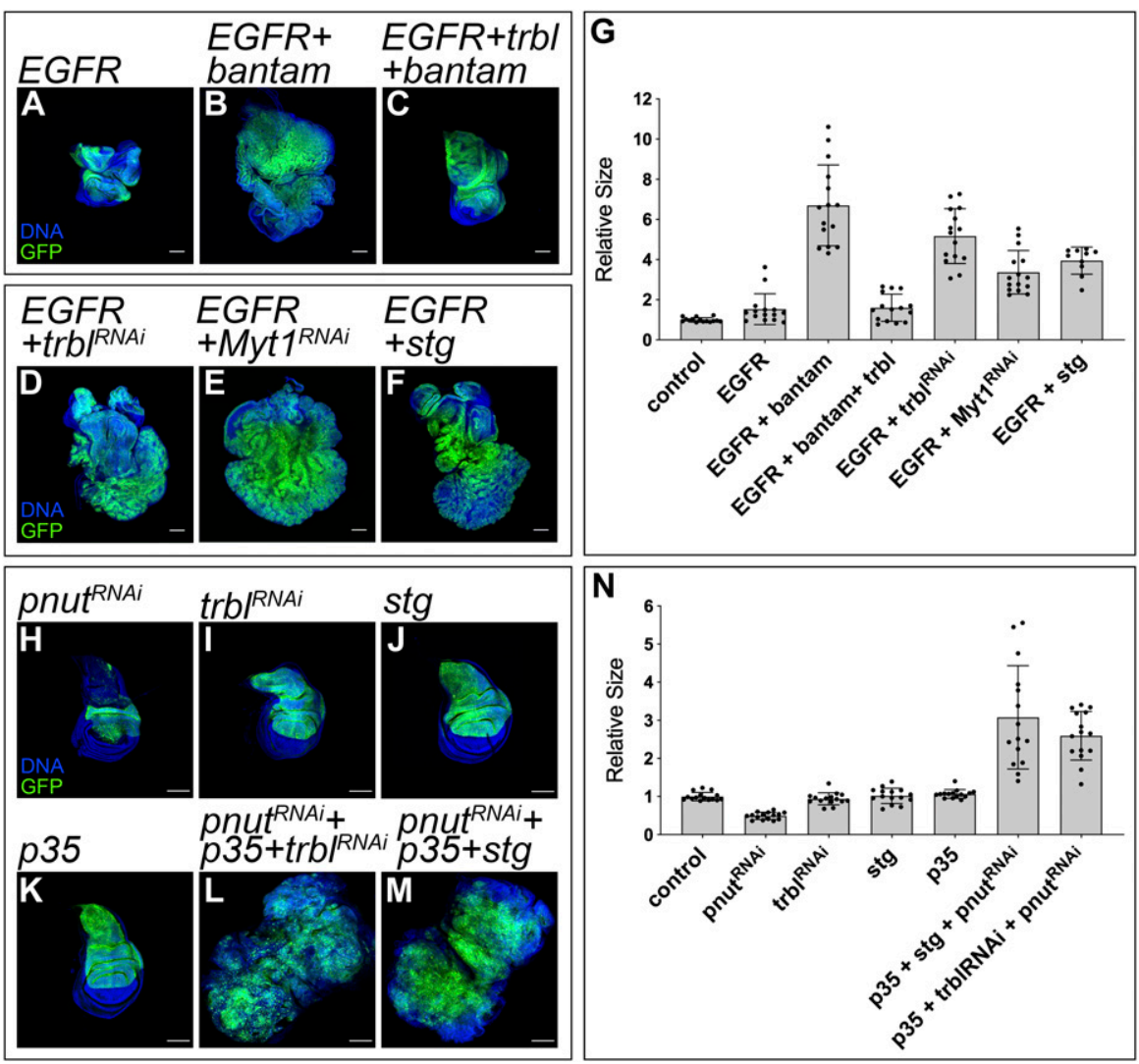

Figure 5. Tumor suppression by trbl.

(A-F) Confocal micrographs of third-instar wing imaginal discs. The indicated genes were expressed under the control of the ap-Gal4 driver (dorsal compartment). The discs also expressed UAS-GFP to label the region where the corresponding transgenes were induced (green). DAPI was used to label the nuclei (blue). ap-Gal4:UAS-GFP was used as control (A). Scale bar: $100 \mu \mathrm{m}$. (G) Quantification of GFP-positive area in third-instar wing imaginal discs. GFP-positive area is normalized to the mean of the control. (H-M) Confocal micrographs of third-instar wing imaginal discs. The indicated genes were expressed under the control of the ap-Gal4 driver (dorsal compartment). The discs also expressed UAS-GFP to label the region where the corresponding transgenes were induced (green). DAPI was used to label the nuclei (blue). Scale bar: $100 \mu \mathrm{m}$. (N) Quantification of GFP-positive area in third-instar wing imaginal discs. GFP-positive area is normalized to the mean of the control. discs with reduced pnut (Gerlach et al, 2018). The oncogenic role of Yki in this context appears to be conserved and expression of YAP, the human ortholog of $y k i$, also triggers tumorigenesis in human cells with cytokinesis failure (Ganem et al, 2014).

The fact that bantam is a central Yki target led us to analyze whether trbl regulation contributes to tumorigenesis in cells knocking down pnut. Wing discs expressing UAS-pnut-RNAi activate the c-Jun $\mathrm{N}$-terminal kinase (JNK) pathway, which mediates their elimination by apoptosis. However, those cells accumulate in G2 and suppression of apoptosis is not sufficient to induce tumorigenesis (Gerlach et al, 2018). We tested whether trbl depletion could trigger tumorigenesis in cells depleting pnut and protected against apoptosis. Remarkably, simultaneous expression of pnut-RNAi, p35, and trbl-RNAi induced the formation of disorganized organs resembling tumors (Fig $5 \mathrm{H}-\mathrm{N}$ ). Comparable results were obtained when we expressed cdc25-stg as an alternative means of driving G2/M transition (Fig $5 \mathrm{M}$ and $\mathrm{N}$ ). The expression of $p 35$ on its own did not affect tissue growth (Fig 5 K and N).

In conclusion, we provide evidence showing that trbl down-regulation fuels tumorigenesis in two independent scenarios: EGFR-driven tumors, and discs depleting pnut and protected against apoptosis.

\section{Discussion}

Here, we identify the cell cycle regulator trbl as a direct bantam target gene involved in the growth regulatory role of this miRNA.
Although trbl knock down accelerates G2/M progression, this does not produce obvious changes in tissue size. Two mechanisms might explain this apparent discrepancy. (1) Cells in the wing disc compensate for perturbations in the length of specific phases of the cell cycle by altering the duration of the other phases. This serves as a mechanism to keep normal division rates of cell proliferation, even when certain phases of the cycle are affected (Reis \& Edgar, 2004). Faster G2/M progression, as a consequence of trbl depletion, would slow down G1/ S to maintain a normal rate of cell division. (2) Perturbations in the cell cycle are normally accompanied by an increase in cell death (Karim \& Rubin, 1998; Neufeld et al, 1998). Consistently, apoptosis can be detected in discs with reduced trbl, which might serve as a mechanism to prevent tissue overgrowth and tumor formation. Furthermore, knock down of the proapoptotic bantam target hid, combined with trbl depletion, induces tissue hyperplasia. Our results establish the minimal combination of bantam targets that, when depleted, drives tissue overgrowth. Tumors commonly combine increased rates of cell division with suppression of apoptosis (Green \& Evan, 2002). We provide a new example, whereby defects in the cell cycle and apoptosis lead to tissue hyperplasia. In addition to trbl and hid, bantam represses other negative growth regulators (Brennecke et al, 2003; Herranz \& Cohen, 2010; Herranz et al., 2012a, 2012b). Although individual depletion of those elements does not result in tissue hyperplasia, we cannot exclude them as important bantam targets in growth control that, together with trbl and hid, might contribute to the growth-promoting role of bantam. 
The repressor of cytokine signaling, Socs36E, is one of the bantam target genes identified so far. bantam cooperates with EGFR in the formation of metastatic tumors, and Socs36E plays a central role in that process. Socs36E down-regulation causes an increase in JAK/STAT pathway activity that, in combination with EGFR, drives malignancy (Herranz et al, 2012b; Hombria \& Serras, 2013). SOCS5 is the Socs36E human ortholog and, as observed in flies, SOCS5 acts as a tumor suppressor in EGFR/RAS-dependent cell transformation assays (Herranz et al, 2012b). Subsequent analyses found that the transforming activity of oncogenic Ras-V12 relies on its ability to down-regulate SOCS5/6 in human cell lines (Hong et al, 2014). The results presented here show that trbl limits the magnitude of growth of tumors co-expressing EGFR and bantam, and trbl down-regulation potentiates EGFR-driven tumor formation. This effect does not appear to be unique for EGFR-driven tumors and trbl is also required to limit tumorigenesis in cells with defective cytokinesis.

Cytokinesis failure can be tumorigenic, and it is proposed that around $40 \%$ of all human cancers have undergone a genome duplication event during their evolution (Zack et al, 2013). In Drosophila, cells with cytokinesis failure are eliminated by apoptosis as a consequence of JNK activation. Suppression of apoptosis is not sufficient to promote tumorigenesis as those cells accumulate in G2 and proliferate poorly (Gerlach et al, 2018). Additional signals are required to induce proliferation and tumorigenesis. Here, we show that trbl is one of those elements, and trbl depletion sufficed to trigger tumorigenesis in cells with cytokinesis failure protected against apoptosis.

trbl down-regulation is sufficient to drive tumorigenesis in wing discs expressing the oncogene EGFR. However, discs with cleavage defects also require suppression of apoptosis for tumor formation. Interestingly, the EGFR pathway promotes cell survival through hid down-regulation by Ras (Bergmann et al, 1998; Kurada \& White, 1998). The role of EGFR in cell survival might explain why EGFRdriven tumors are not dependent on additional blockage of apoptosis, whereas discs down-regulating pnut and trbl require suppression of apoptosis for tumor formation. Studying whether Trbl plays similar roles in human tumor models might merit further investigation.

Previous studies have shown that different growth regulators such as Ras, dMyc, and dPI3K control tissue growth by regulating G1/S progression (Johnston et al, 1999; Weinkove et al, 1999; Prober \& Edgar, 2000, 2002). Yki also controls G1/S progression by regulating the expression of genes, including Cyclin E, E2F, and dMyc (Wu et al, 2003; Goulev et al, 2008; Neto-Silva et al, 2010). In addition to that, Yki drives $\mathrm{G} 2 / \mathrm{M}$ progression by regulating the transcription of the phosphatase cdc25-stg (Gerlach et al, 2018). miRNAs are typically embedded in regulatory loops to reduce noise and provide robustness to biological systems (Herranz \& Cohen, 2010). Our results suggest the presence of a feed forward loop (FFL) downstream of Yki that includes the miRNA bantam and modulates G2/M transition. FFLs involve at least three elements: a regulator, $X(Y k i)$, which regulates $Y$ (bantam), and gene $Z$ (cdc25-stg), which is regulated by both $X$ (Yki) and $Y$ (bantam) (Fig 6). Both branches act in the same direction on $Z$ (cdc25-stg), hence representing a coherent FFL (Alon, 2007). Yki drives tissue growth by inducing proliferation and repressing apoptosis. Interestingly, a

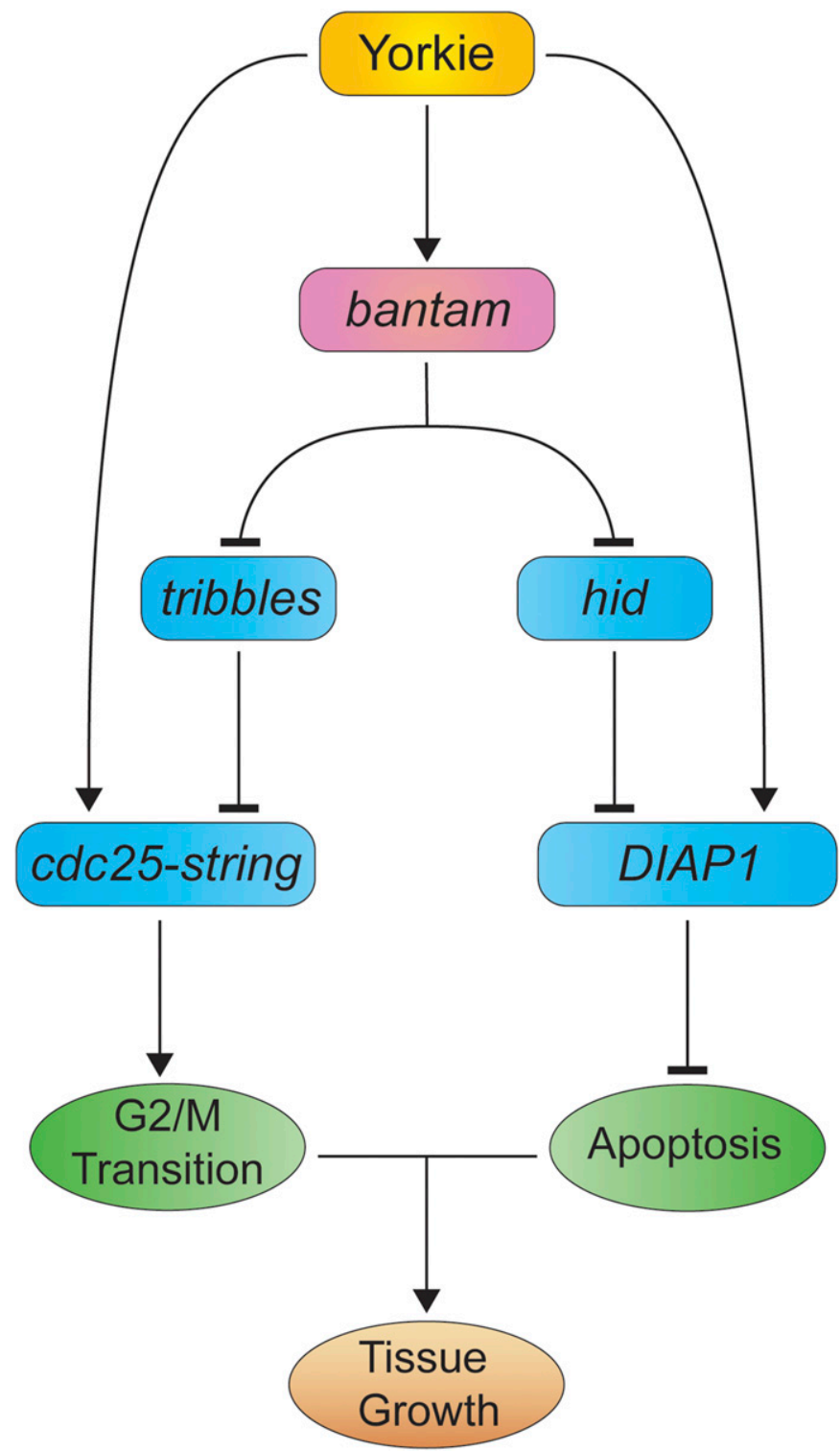

Figure 6. Model illustrating the growth-promoting role of Yki and bantam in the wing epithelium.

Yki promotes growth by inducing cell cycle progression and repressing apoptosis. Yki regulates G2/M progression directly by inducing the expression of its target gene cdc25-stg. Likewise, it limits apoptosis by inducing the expression of the repressor of apoptosis DIAP1. In parallel, Yki induces the expression of the miRNA bantam, which, in turn, represses trbl and hid. trbl acts as an inhibitor of cdc25-stg, and hid represses DIAP1. Yki triggers an FFL controlling cell cycle progression and apoptosis directly, and indirectly through bantam. The combination of these factors leads to tissue growth.

similar coherent FFL involving bantam is present in the "antiapoptotic branch." Yki represses apoptosis directly by inducing the expression of DIAP1, and indirectly by inducing the expression of bantam that in turn represses the proapoptotic gene hid (Fig 6). These types of circuits can be used to reduce noise and ensure developmental decisions leading to an "all-or-none" outcome (Herranz \& Cohen, 2010). This type of loop can serve to drive tumorigenesis, whereby sustained Yki activity would lead to the 
activation of both arms in the FFL, resulting in an excess of proliferation and reduced apoptosis and consequently tumor formation. In fact, these kinds of loops have also been reported operating in human cancer (Iliopoulos et al, 2009).

\section{Materials and Methods}

\section{Drosophila strains}

The following Drosophila strains are described in the cited references: ap-Gal4 and nub-Gal4 (Calleja et al, 1996); ptc-Gal4 (Wilder \& Perrimon, 1995); MS1096-Gal4 (Capdevila \& Guerrero, 1994); bantam$\triangle 1$, UAS-bantam-A, and UAS-bantam-D (Brennecke et al, 2003); EP3622 (Hipfner et al, 2002); UAS-miR-RHG (Siegrist et al, 2010); UASfly-FUCCI (Zielke et al, 2014); UAS-yki (Huang et al, 2005); UAS-EGFR (Buff et al, 1998); UAS-bantam-sponge and bantam-lacZ (P\{lacW\} banL1170a) (Herranz et al, 2012a); FRT-wtsX1 (Xu et al, 1995). Other stocks are described in FlyBase.

The following stocks were provided by the Bloomington Drosophila Stock Center (BDSC): UAS-p35 (\#6298); UAS-stg (\#56562); UAS-pnut-RNAi (\#11791); UAS-Myt1-RNAi (\#62892); and trbl-GFP (\#61654).

The following stocks were provided by the Vienna Drosophila RNAi Center (VDRC): UAS-trbl-RNAi (\#22114); UAS-hid-RNAi (\#8269); and UAS-trbl-RNAi-KK (\#106774).

The complete list of genotypes can be found as Supplemental Data 1.

\section{Transgene expression in the wing imaginal disc}

The apterous and patched enhancers were used to express Gal4 in the wing imaginal disc. To prevent early lethality due to transgene expression, the tubulin-Gal80TS construct was used for gene manipulation experiments. It allows the control of the timing of UAS transgene expression in a temperature dependent manner (Zeidler et al, 2004). Drosophila crosses were kept for 2 days at $18^{\circ} \mathrm{C}$ to lay eggs and larvae were kept for five additional days at $18^{\circ} \mathrm{C}$. After that, the larvae were transferred to $29^{\circ} \mathrm{C}$ to induce transgene expression. Only third-instar wandering larvae were dissected to ensure that the animals analyzed were in the same developmental stage. Figure legends indicate when the timing and temperature differed from this procedure.

\section{trbl 3'UTR sensor}

The trbl 3'UTR was amplified from cDNA (DGRC clone RH69304) with the primers wt-fwd and wt-rev and cloned into the tub-EGFP pCaSpeR4 sensor vector as an Xhol-Notl fragment. We generated a mutation in the bantam $3^{\prime}$ site, via PCR using the wt-fwd and 3'$m t$-rev primers, exchanging TC with AG. To mutate the bantam $5^{\prime}$ site (located more upstream in the trbl $3^{\prime}$ UTR), we performed two PCRs with (1) the wt-fwd and 5' $m$ t-rev primers, exchanging AT with GC and (2) the $5^{\prime} m t$-fwd and wt-rev primers, again exchanging AT with GC. We generated the full trbl 3'UTR with the bantam 5' site mutated in a subsequent PCR using these overlapping fragments as templates together with the wt-fwd and wt-rev primers. Using the same strategy but combining the wt-fwd-with the $3^{\prime}$-mt-rev primers, we generated the trbl 3'UTR with both bantam sites mutated.

The following primers were used:

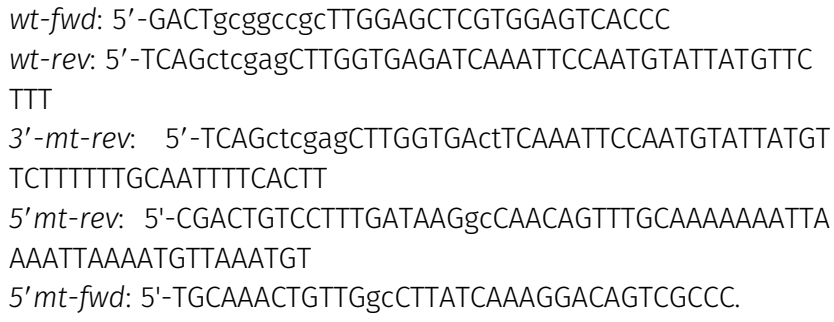

\section{Immunohistochemistry}

The following primary antibodies were used: anti-ß-Gal (Developmental Studies Hybridoma Bank; 40-1a; mouse; 1:20); antiCas3-act (no. 9661; rabbit; 1:100; Cell Signaling Technology); anti-PH3 (no. 9701; rabbit; 1:100; Cell Signaling Technology). The following secondary antibodies were used: antimouse Alexa Fluor 555 (A21425; goat; 1:400; Invitrogen); antimouse Alexa Fluor 647 (A-21237; goat; 1:400; Invitrogen); anti-rabbit Alexa Fluor 555 (A-21430; goat; 1: 400; Invitrogen); and antirabbit Alexa Fluor 647 (A-21246; goat; 1:400; Invitrogen).

Third-instar larvae were dissected in PBS. Fixation of the sample was performed with $4 \%$ formaldehyde solution for $20 \mathrm{~min}$ at room temperature. After that, the samples were washed three times for 10 min in phosphate-buffered saline-tween (PBT) and blocked for 20 min in PBT-BSA (BBT). The first antibody diluted in BBT was incubated with the samples at room temperature overnight followed by three 15-min washes in BBT. The secondary antibody together with DAPI in BBT was incubated with the sample for $2 \mathrm{~h}$ at room temperature followed by four 15 -min washes in PBT. The samples were mounted in $90 \%$ glycerol with PBS containing 0.05\% propyl gallate. Images of wing imaginal discs were acquired with a Leica SP8 confocal laser-scanning microscope and processed with ImageJ and Adobe Photoshop CC.

\section{Genetic mosaics}

The FLP/FRT technique (Xu \& Rubin, 1993) was used to generate bantam and wts loss of function clones. Second-instar larvae were heat-shocked for $1 \mathrm{~h}$ at $37^{\circ} \mathrm{C}$. Next, the larvae were kept at $25^{\circ} \mathrm{C}$. Clones were visualized by either loss of RFP or $\beta$-Gal expression.

\section{Flow cytometry}

Dissection of wing imaginal discs of third-instar larvae was performed in PBS and trypsin-EDTA solution was used to dissociate the sample for $45 \mathrm{~min}$. Cells were fixed with $4 \%$ formaldehyde solution for $20 \mathrm{~min}$ and stored in $70 \%$ ethanol for $2 \mathrm{~h}$. The samples were incubated with DAPI in PBT overnight at $4^{\circ} \mathrm{C}$. Flow cytometry was used to measure fluorescence of DAPI, GFP, and RFP in cells with a BD FACSAria Fusion. Doublet cells were excluded with an integral/ peak dot plot of DAPI fluorescence. 


\section{Adult wing size and cell number quantification}

The MS1096 and nubbin enhancers were used to express Gal4 in the wing. Adult flies were fixed in glycerol-ethanol (25\% glycerol, $75 \%$ ethanol) for three days and the wings were mounted in glycerol for imaging. Wing images of adult flies were taken with a Leica DM 5500B bright-field microscope. Tissue area measurement was performed with ImageJ. Bristle counting to determine cell density was obtained from high-magnification pictures of the region immediately anterior to the cross vein between veins four and five. The number of bristles in a $480 \times 170$ pixels area was quantified, and unpaired $t$ test was used to determine statistical significance. Graphs were generated with GraphPad Prism 7.

\section{Wing imaginal disc size quantification}

Eggs were collected on apple agar plates with yeast paste at $25^{\circ} \mathrm{C}$ for $24 \mathrm{~h}$ and larvae were kept on the plates for another $24 \mathrm{~h}$. A maximum of 30 larvae were transferred to each vial with standard Drosophila food and stored for 4 days on $18^{\circ} \mathrm{C}$. Then, the vials were switched to $29^{\circ} \mathrm{C}$ to activate transgene expression. Larvae were dissected as third-instar wandering larvae to ensure same developmental timing. Images were acquired with a Leica SP8 confocal laser-scanning microscope. The option "Threshold" was used to mark the whole GFP-positive area in Imagej. After this, the option "Analyze Particles" was used to determine the area size. Unpaired $t$ test was used to determine statistical significance and graphs were generated with GraphPad Prism 7. Figure legends indicate when the timing, temperature, or the fluorophore used for the area quantification differed from this procedure.

\section{Quantification of cells in mitosis}

Third-instar wing imaginal discs were stained against PH3, which specifically labels mitotic cells. Images were acquired of the apical part of the wing disc with a Leica SP8 confocal laser-scanning microscope. An area of $250 \times 250$ pixels was used to count PH3positive nuclei. Unpaired $t$ test was used to determine statistical significance, and graphs were generated with GraphPad Prism 7.

\section{Quantification of apoptosis}

Third-instar wing imaginal discs were stained against Cas3-act, which marks cells undergoing apoptosis. Images were acquired of the basal part of the wing disc with a Leica SP8 confocal laserscanning microscope. An area of $250 \times 250$ pixels in the wing pouch was used to quantify the signal. The overall intensity of Cas3-act per genotype was quantified by using the measurement "Mean Grey Value" in ImageJ. Unpaired t test was used to determine statistical significance, and graphs were generated with GraphPad Prism 7.

\section{Western blot}

15 third-instar larvae were transferred into protein extraction and immunoprecipitation buffer (RIPA; Sigma-Aldrich), complete Protease Inhibitor Cocktail (Roche) added, and the larvae homogenized. The homogenate was centrifuged (15 min, 13,000 rpm,
Eppendorf Centrifuge 5424, Rotor FA-45-24-11), and the supernatant used further. The BCA protein assay (Thermo Fisher Scientific) was used to determine the protein concentration. $10 \mu \mathrm{g}$ of protein was loaded per sample for the Western blots. Antibody against GFP (ab13970, chicken; Abcam) was used to detect Trbl-GFP fusion protein. Antibody against Actin (A5441, mouse; Sigma-Aldrich) was used as a control. Secondary HRP goat antichicken (A16054; Invitrogen) and goat antimouse (P0447; Dako) antibodies were used together with the Pierce ECL Western Blotting Substrate (Thermo Fisher Scientific) to visualize protein levels.

\section{EdU incorporation}

Third-instar larvae were dissected in PBS and incubated in $300 \mathrm{mM}$ EdU in PBS for 30 min. Fixation of the sample was performed with $4 \%$ formaldehyde solution for $20 \mathrm{~min}$ at room temperature. Following, the samples were washed three times for $10 \mathrm{~min}$ in PBT and blocked for 20 min in BBT. The Click-iT EdU Alexa Fluor 488 Imaging Kit (Invitrogen) was used, and the samples were incubated for $1 \mathrm{~h}$ with the reaction mix provided by the Imaging Kit followed by one BBT wash for $10 \mathrm{~min}$. The samples were incubated with DAPI in BBT for $2 \mathrm{~h}$ at room temperature followed by four 15-min washes with PBT. The samples were mounted in $90 \%$ glycerol with PBS containing $0.05 \%$ propyl gallate.

\section{Acknowledgements}

We thank Carlos Estella for reagents; Melissa Alexandra Visser for technical support; Jan Michael Kugler for technical guidance; Olive Benét Kersey for comments on the manuscript; and the DSHB, VDRC, and BDSC for antibodies and fly strains. This work was supported by Novo Nordisk Foundation (grant number NNF0052223), a grant by the Neye Foundation for genetic models for cancer gene discovery, and a grant by Læge Sofus Carl Emil Friis og Hustru Olga Doris Friis' Legat.

\section{Author Contributions}

SU Gerlach: conceptualization, data curation, formal analysis, validation, investigation, visualization, methodology, and writing-original draft, review, and editing.

M Sander: data curation, formal analysis, investigation, methodology, and writing-review and editing.

S Song: conceptualization, formal analysis, investigation, visualization, and methodology.

H Herranz: conceptualization, resources, supervision, funding acquisition, investigation, methodology, project administration, and writing-original draft, review, and editing.

\section{Conflict of Interest Statement}

The authors declare that they have no conflict of interest.

\section{References}

Alon U (2007) Network motifs: Theory and experimental approaches. Nat Rev Genet 8: 450-461. doi:10.1038/nrg2102 
Becam I, Rafel N, Hong X, Cohen SM, Milan M (2011) Notch-mediated repression of bantam miRNA contributes to boundary formation in the Drosophila wing. Development 138: 3781-3789. doi:10.1242/ dev.064774

Bergmann A, Agapite J, McCall K, Steller H (1998) The Drosophila gene hid is a direct molecular target of Ras-dependent survival signaling. Cell 95: 331-341. doi:10.1016/s0092-8674(00)81765-1

Brand AH, Perrimon N (1993) Targeted gene expression as a means of altering cell fates and generating dominant phenotypes. Development 118: 401-415.

Brennecke J, Hipfner DR, Stark A, Russell RB, Cohen SM (2003) bantam encodes a developmentally regulated microRNA that controls cell proliferation and regulates the proapoptotic gene hid in Drosophila. Cell 113: 25-36. doi:10.1016/s0092-8674(03)00231-9

Buff E, Carmena A, Gisselbrecht S, Jimenez F, Michelson AM (1998) Signalling by the Drosophila epidermal growth factor receptor is required for the specification and diversification of embryonic muscle progenitors. Development 125: 2075-2086.

Calleja M, Moreno E, Pelaz S, Morata G (1996) Visualization of gene expression in living adult Drosophila. Science 274: 252-255. doi:10.1126/ science.274.5285.252

Capdevila J, Guerrero I (1994) Targeted expression of the signaling molecule decapentaplegic induces pattern duplications and growth alterations in Drosophila wings. EMBO / 13: 4459-4468. doi:10.1002/j.14602075.1994.tb06768.x

Carthew RW, Agbu P, Giri R (2017) MicroRNA function in Drosophila melanogaster. Semin Cell Dev Biol 65: 29-37. doi:10.1016/ j.semcdb.2016.03.015

Conlon I, Raff M (1999) Size control in animal development. Cell 96: 235-244. doi:10.1016/s0092-8674(00)80563-2

Das R, Sebo Z, Pence L, Dobens LL (2014) Drosophila tribbles antagonizes insulin signaling-mediated growth and metabolism via interactions with Akt kinase. PLoS One 9: e109530. doi:10.1371/journal.pone.0109530

Esquela-Kerscher A, Slack FJ (2006) Oncomirs: microRNAs with a role in cancer. Nat Rev Cancer 6: 259-269. doi:10.1038/nrc1840

Ganem NJ, Cornils H, Chiu SY, O'Rourke KP, Arnaud J, Yimlamai D, Thery M, Camargo FD, Pellman D (2014) Cytokinesis failure triggers hippo tumor suppressor pathway activation. Cell 158: 833-848. doi:10.1016/ j.cell.2014.06.029

Ganem NJ, Storchova Z, Pellman D (2007) Tetraploidy, aneuploidy and cancer. Curr Opin Genet Dev 17: 157-162. doi:10.1016/j.gde.2007.02.011

Gerlach SU, Eichenlaub T, Herranz H (2018) Yorkie and JNK control tumorigenesis in Drosophila cells with cytokinesis failure. Cell Rep 23: 1491-1503. doi:10.1016/j.celrep.2018.04.006

Gonzalez C (2013) Drosophila melanogaster: A model and a tool to investigate malignancy and identify new therapeutics. Nat Rev Cancer 13: 172-183. doi:10.1038/nrc3461

Goulev Y, Fauny JD, Gonzalez-Marti B, Flagiello D, Silber J, Zider A (2008) SCALLOPED interacts with YORKIE, the nuclear effector of the hippo tumor-suppressor pathway in Drosophila. Curr Biol 18: 435-441. doi:10.1016/j.cub.2008.02.034

Green DR, Evan GI (2002) A matter of life and death. Cancer Cell 1: 19-30. doi:10.1016/s1535-6108(02)00024-7

Hanahan D, Weinberg RA (2011) Hallmarks of cancer: The next generation. Cell 144: 646-674. doi:10.1016/j.cell.2011.02.013

Hariharan IK (2015) Organ size control: Lessons from Drosophila. Dev Cell 34: 255-265. doi:10.1016/j.devcel.2015.07.012

Hay BA, Wolff T, Rubin GM (1994) Expression of baculovirus P35 prevents cell death in Drosophila. Development 120: 2121-2129.

Herranz H, Cohen SM (2010) MicroRNAs and gene regulatory networks: Managing the impact of noise in biological systems. Genes Dev 24: 1339-1344. doi:10.1101/gad.1937010
Herranz H, Eichenlaub T, Cohen SM (2016) Cancer in Drosophila: Imaginal discs as a model for epithelial tumor formation. Curr Top Dev Biol 116: 181-199. doi:10.1016/bs.ctdb.2015.11.037

Herranz H, Hong X, Cohen SM (2012a) Mutual repression by bantam miRNA and Capicua links the EGFR/MAPK and Hippo pathways in growth control. Curr Biol 22: 651-657. doi:10.1016/j.cub.2012.02.050

Herranz H, Hong X, Hung NT, Voorhoeve PM, Cohen SM (2012b) Oncogenic cooperation between SOCS family proteins and EGFR identified using a Drosophila epithelial transformation model. Genes Dev 26: 1602-1611. doi:10.1101/gad.192021.112

Herranz H, Hong X, Perez L, Ferreira A, Olivieri D, Cohen SM, Milan M (2010) The miRNA machinery targets Mei-P26 and regulates Myc protein levels in the Drosophila wing. EMBO J 29: 1688-1698. doi:10.1038/ emboj.2010.69

Hipfner DR, Weigmann K, Cohen SM (2002) The bantam gene regulates Drosophila growth. Genetics 161: 1527-1537.

Hombria JC, Serras F (2013) Why should we care about fly tumors?: The case of JAK-STAT and EGFR cooperation in oncogenesis. JAKSTAT 2: e23203. doi:10.4161/jkst.23203

Hong SH, Kang M, Lee KS, Yu K (2016) High fat diet-induced TGF-beta/Gbb signaling provokes insulin resistance through the tribbles expression. Sci Rep 6: 30265. doi:10.1038/srep30265

Hong X, Nguyen HT, Chen Q, Zhang R, Hagman Z, Voorhoeve PM, Cohen SM (2014) Opposing activities of the Ras and Hippo pathways converge on regulation of YAP protein turnover. EMBO J 33: 2447-2457. doi:10.15252/ embj.201489385

Huang J, Wu S, Barrera J, Matthews K, Pan D (2005) The Hippo signaling pathway coordinately regulates cell proliferation and apoptosis by inactivating Yorkie, the Drosophila Homolog of YAP. Cell 122: 421-434. doi:10.1016/j.cell.2005.06.007

Iliopoulos D, Hirsch HA, Struhl K (2009) An epigenetic switch involving NFkappaB, Lin28, Let-7 MicroRNA, and IL6 links inflammation to cell transformation. Cell 139: 693-706. doi:10.1016/j.cell.2009.10.014

Jin Z, Homola E, Tiong S, Campbell SD (2008) Drosophila myt1 is the major cdk1 inhibitory kinase for wing imaginal disc development. Genetics 180: 2123-2133. doi:10.1534/genetics.108.093195

Johnston LA, Edgar BA (1998) Wingless and Notch regulate cell-cycle arrest in the developing Drosophila wing. Nature 394: 82-84. doi:10.1038/ 27925

Johnston LA, Prober DA, Edgar BA, Eisenman RN, Gallant P (1999) Drosophila myc regulates cellular growth during development. Cell 98: 779-790. doi:10.1016/s0092-8674(00)81512-3

Karim FD, Rubin GM (1998) Ectopic expression of activated Ras1 induces hyperplastic growth and increased cell death in Drosophila imaginal tissues. Development 125: 1-9.

Kurada P, White K (1998) Ras promotes cell survival in Drosophila by downregulating hid expression. Cell 95: 319-329. doi:10.1016/s00928674(00)81764-X

Loya CM, Lu CS, Van Vactor D, Fulga TA (2009) Transgenic microRNA inhibition with spatiotemporal specificity in intact organisms. Nat Methods 6: 897-903. doi:10.1038/nmeth.1402

Mata J, Curado S, Ephrussi A, Rorth P (2000) Tribbles coordinates mitosis and morphogenesis in Drosophila by regulating string/CDC25 proteolysis. Cell 101: 511-522. doi:10.1016/s0092-8674(00)80861-2

Morata G, Ripoll P (1975) Minutes: Mutants of drosophila autonomously affecting cell division rate. Dev Biol 42: 211-221. doi:10.1016/00121606(75)90330-9

Neto-Silva RM, de Beco S, Johnston LA (2010) Evidence for a growthstabilizing regulatory feedback mechanism between Myc and Yorkie, the Drosophila homolog of Yap. Dev Cell 19: 507-520. doi:10.1016/ j.devcel.2010.09.009 
Neufeld TP, de la Cruz AF, Johnston LA, Edgar BA (1998) Coordination of growth and cell division in the Drosophila wing. Cell 93: 1183-1193. doi:10.1016/s0092-8674(00)81462-2

Neufeld TP, Rubin GM (1994) The Drosophila peanut gene is required for cytokinesis and encodes a protein similar to yeast putative bud neck filament proteins. Cell 77: 371-379. doi:10.1016/0092-8674(94)90152-x

Nolo R, Morrison CM, Tao C, Zhang X, Halder G (2006) The bantam microRNA is a target of the hippo tumor-suppressor pathway. Curr Biol 16: 1895-1904. doi:10.1016/j.cub.2006.08.057

Pan D (2010) The hippo signaling pathway in development and cancer. Dev Cell 19: 491-505. doi:10.1016/j.devcel.2010.09.011

Prober DA, Edgar BA (2000) Ras1 promotes cellular growth in the Drosophila wing. Cell 100: 435-446. doi:10.1016/s0092-8674(00)80679-0

Prober DA, Edgar BA (2002) Interactions between Ras1, dMyc, and dPI3K signaling in the developing Drosophila wing. Genes Dev 16: 2286-2299. doi:10.1101/gad.991102

Reis T, Edgar BA (2004) Negative regulation of dE2F1 by cyclin-dependent kinases controls cell cycle timing. Cell 117: 253-264. doi:10.1016/s00928674(04)00247-8

Sakaue-Sawano A, Kurokawa H, Morimura T, Hanyu A, Hama H, Osawa H, Kashiwagi S, Fukami K, Miyata T, Miyoshi H, et al (2008) Visualizing spatiotemporal dynamics of multicellular cell-cycle progression. Cell 132: 487-498. doi:10.1016/j.cell.2007.12.033

Siegrist SE, Haque NS, Chen CH, Hay BA, Hariharan IK (2010) Inactivation of both Foxo and reaper promotes long-term adult neurogenesis in Drosophila. Curr Biol 20: 643-648. doi:10.1016/j.cub.2010.01.060

Thompson BJ, Cohen SM (2006) The Hippo pathway regulates the bantam microRNA to control cell proliferation and apoptosis in Drosophila. Cell 126: 767-774. doi:10.1016/j.cell.2006.07.013

Venken KJ, Schulze KL, Haelterman NA, Pan H, He Y, Evans-Holm M, Carlson JW, Levis RW, Spradling AC, Hoskins RA, et al (2011) MiMIC: A highly versatile transposon insertion resource for engineering Drosophila melanogaster genes. Nat Methods 8: 737-743. doi:10.1038/nmeth.1662
Vivanco I, Sawyers CL (2002) The phosphatidylinositol 3-Kinase AKT pathway in human cancer. Nat Rev Cancer 2: 489-501. doi:10.1038/nrc839

Weinkove D, Neufeld TP, Twardzik T, Waterfield MD, Leevers SJ (1999) Regulation of imaginal disc cell size, cell number and organ size by Drosophila class I(A) phosphoinositide 3-kinase and its adaptor. Curr Biol 9: 1019-1029. doi:10.1016/s0960-9822(99)80450-3

Wilder EL, Perrimon N (1995) Dual functions of wingless in the Drosophila leg imaginal disc. Development 121: 477-488.

Wu S, Huang J, Dong J, Pan D (2003) hippo encodes a Ste-20 family protein kinase that restricts cell proliferation and promotes apoptosis in conjunction with salvador and warts. Cell 114: 445-456. doi:10.1016/ s0092-8674(03)00549-x

Xu T, Rubin GM (1993) Analysis of genetic mosaics in developing and adult Drosophila tissues. Development 117: 1223-1237.

Xu T, Wang W, Zhang S, Stewart RA, Yu W (1995) Identifying tumor suppressors in genetic mosaics: The Drosophila lats gene encodes a putative protein kinase. Development 121: 1053-1063.

Zack TI, Schumacher SE, Carter SL, Cherniack AD, Saksena G, Tabak B, Lawrence MS, Zhsng CZ, Wala J, Mermel CH, et al (2013) Pan-cancer patterns of somatic copy number alteration. Nat Genet 45: 1134-1140. doi:10.1038/ng.2760

Zeidler MP, Tan C, Bellaiche Y, Cherry S, Hader S, Gayko U, Perrimon N (2004) Temperature-sensitive control of protein activity by conditionally splicing inteins. Nat Biotechnol 22: 871-876. doi:10.1038/nbt979

Zielke N, Korzelius J, van Straaten M, Bender K, Schuhknecht GF, Dutta D, Xiang J, Edgar BA (2014) Fly-FUCCl: A versatile tool for studying cell proliferation in complex tissues. Cell Rep 7: 588-598. doi:10.1016/ j.celrep.2014.03.020

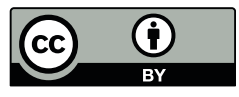

License: This article is available under a Creative Commons License (Attribution 4.0 International, as described at https://creativecommons.org/ licenses/by/4.0/). 